\title{
Nonlinear observer to estimate polarization phenomenon in membrane distillation
}

\author{
Billal Khoukhi*, Mohamed Tadjine, and Mohamed Seghir Boucherit \\ Process Control Laboratory, Control Engineering Department, Ecole Nationale Polytechnique, 10 avenue Pasteur, \\ Hassan-Badi, 16200 Algiers, Algeria
}

Received 3 April 2015 / Accepted 21 August 2015

\begin{abstract}
This paper presents a bi-dimensional dynamic model of Direct Contact Membrane Desalination (DCMD) process. Most of the MD configuration processes have been modeled as steady-state one-dimensional systems. Stationary two-dimensional MD models have been considered only in very few studies. In this work, a dynamic model of a DCMD process is developed. The model is implemented using Matlab/Simulink environment. Numerical simulations are conducted for different operational parameters at the module inlets such as the feed and permeate temperature or feed and permeate flow rate. The results are compared with experimental data published in the literature. The work presents also a feed forward control that compensates the possible decrease of the temperature gradient by increasing the flow rate. This work also deals with a development of nonlinear observer to estimate temperature polarization inside the membrane. The observer gives a good profile and longitudinal temperature estimations and shows a good prediction of pure water flux production.
\end{abstract}

Key words: Direct contact membrane distillation, Dynamic modeling, Heat and mass transfer, Unknown input observer, Polarization coefficient, Saline water desalination.

\section{Introduction}

Membrane distillation (MD) process is an emerging technology for water treatment. The driving force of the MD process is given by the pressure difference of vapor formed by a difference in temperature of solutions on both sides of a hydrophobic membrane [1]. The advantages of Direct Contact Membrane Desalination (DCMD) lie in its simplicity, the need for only small temperature differences and nearly $100 \%$ rejection of dissolved solids [1]. Furthermore, the low energy demand systems in DCMD processes can be equipped with renewable energy equipment such as solar collectors [2] and solar distillers [3]. Many MD configuration processes have been modeled as steady-state one-dimensional systems using empirical heat and mass transfer equations [4]. Only few publications use stationary one or two-dimensional heat-transfer equations to simulate the process more accurately. Although many semiempirical models have been developed, a detailed model for temperature polarization on flat-plate MD processes is still lacking. Some studies were interested in a theoretical modeling and experimental analysis of direct contact membrane distillation in steady state such as in [4]. A dynamic modeling of

*e-mail: khoukhi.billal@gmail.com direct contact membrane distillation processes has also been presented in $[5,6]$. In another way, to show the interest of using renewable energies in DCMD processes, a performance investigation of a solar-assisted direct contact membrane distillation system was conducted in [7].

Our study focuses on a bi dimensional dynamic model to simulate the membrane temperature and the pure water flux. It proposes an algorithm to resolve the temperature partial differential equations (PDE) that describes the process.

Our study also uses a nonlinear observer in order to estimate all temperatures and fluxes inside the membrane from the measurement of the accessible data of the membrane, which are inlet and outlet temperatures.

Because temperature inside the membrane is not accessible for measurement this observer is very useful and can be considered as a software sensor in order to estimate temperature distribution.

Several approaches have been tried to extend the Unknown Input Observer (UIO) design for linear systems to nonlinear systems. The first works have been dedicated to UIOs for bilinear systems [8]. Other approaches based on the transformation of the nonlinear system into canonical forms are proposed in [9] and [10]. The limitation of these approaches is that the 


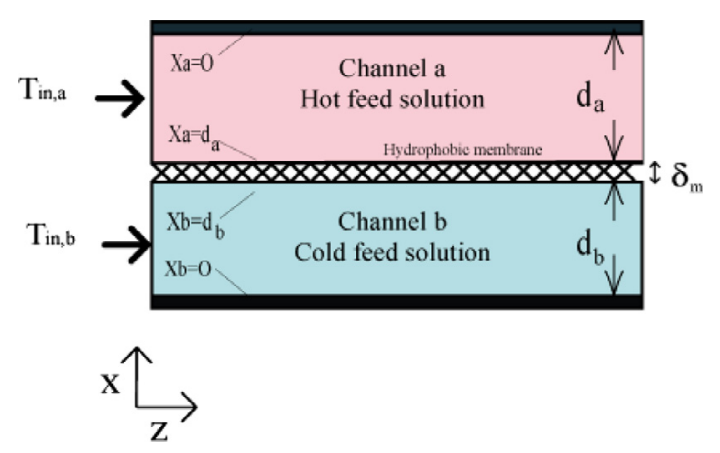

Figure 1. Schematic diagram of membrane distillation process.

required state transformation does not exist for all nonlinear systems but only for a limited class. The observer developed in this paper is designed in a cascade structure and is specific to the model of DCMD.

This paper is organized as follow: in the next section a model of the DCMD is presented. In Section 3 a nonlinear observer is developed. In Section 4 a presentation of the simulation results is given. Finally, the paper close with some concluding remarks.

\section{Modeling the membrane distillation process}

In Direct Contact Membrane Distillation (DCMD) both sides of the membrane are in direct contact with a liquid stream. On the left of the membrane shown in Figure 1, the hot liquid (i.e. hot seawater) flows in the evaporator channel, whilst on the right a cold liquid (i.e. cooled permeate or distillate) is circulated. Heat transfer (as well as mass transfer) occurs from the hotter to the colder side. The liquid in the evaporator channel is constantly refilled and reheated, whilst the volume of the liquid in the permeate channel increases and heats up. One of the main features of DCMD is that the gas gap between the membrane surface and the condensate stream is very narrow and only exists due to the hydrophobic nature of the membrane. This causes the temperature of the membrane surface in contact with the condensate to be very close to that of the condensate stream itself, thus allowing high temperature drops across the membrane, i.e. high driving forces for mass transfer. Conversely, the Direct Contact configuration causes a relatively high heat loss, as the membrane is the only barrier for the transfer of sensible heat [2]. In the following, we will give the mathematical equations that describe those phenomena.

\subsection{Pure water flux in the distillation process}

The mass transfer driving force across the membrane is the difference in saturated pressure components on both membrane surfaces due to the temperature gradient. The general mass flux form can be expressed as follows:

$$
J=c_{m} \Delta P^{\text {sat }}=c_{m}\left(P_{a}^{\text {sat }}-P_{b}^{\text {sat }}\right)
$$

where $\Delta P^{\text {sat }}=P_{a}^{\text {sat }}-P_{b}^{\text {sat }}$ and $P_{1}^{\text {sat }}, P_{2}^{\text {sat }}$ are the saturated pressure of water on the hot and cold feed membrane surfaces, respectively. For non-ideal binary mixtures, the flux can be determined as $[4,11]$ :

$$
J=c_{m}\left(\left(1-x_{\mathrm{NaCl}}\right)\left(1-0.5 x_{\mathrm{NaCl}}-10 x_{\mathrm{Nacl}}^{2}\right) P_{a}^{\mathrm{sat}}-P_{b}^{\mathrm{sat}}\right)
$$

where $x_{\mathrm{NaCl}}$ is the mole fraction of $\mathrm{NaCl}$ in saline solution. Saturated pressures can be determined by the Antoine equation [12]:

$$
P_{k}^{\text {sat }}=133.322 \times 10^{\left(8.10-\left(1450 /\left(T_{k}+235\right)\right)\right)}, \quad k=a, b .
$$

where $T_{k}$ is the temperature in ${ }^{\circ} \mathrm{C}$. The membrane coefficient $c_{m}$ in (1) can be estimated by a weighted sum (via parameters $\alpha(T)$ and $\beta(T))$ of the Knudsen diffusion and the Poiseuille (viscous) flow models [13], i.e.

$$
\begin{aligned}
c_{m} & =c_{k}+c_{p} \\
& =1.064 \alpha(T) \frac{\varepsilon r}{\tau \delta_{m}} \sqrt{\frac{M_{w}}{R T_{m}}}+0.125 \beta(T) \frac{\varepsilon r^{2}}{\tau \delta_{m}} \frac{M_{w} P_{m}}{\eta_{v} R T_{m}}
\end{aligned}
$$

where, $M_{w}$ is the molecular weight of water, $P_{m}$ is the mean saturated pressure in membrane, $R$ is the gas constant, $r$ is the pore radius of the membrane, $T_{m}$ is the mean temperature in membrane, $\delta_{m}$ is the thickness of membrane, $\varepsilon$ is the porosity of membrane, $\eta_{v}$ is the gas viscosity and $\tau$ is the tortuosity factor. The tortuosity of a porous hydrophobic membrane was estimated by [14].

The objective of the modeling is to estimate the temperature distribution in the flow channels and use the equation (2) to calculate the pure water flux.

\subsection{The temperature equations in the flow channel}

Figure 1 shows a schematic diagram of the parallel-flow membrane distillation system. With a hydrophobic, micro porous membrane, liquid water is prevented from entering the pores, while molecular water in the vapor phase can pass through. In order to obtain accurate temperature distribution on membrane surfaces with position, a study of heat transfer and transport phenomena should be done $[15,16]$.

The three-dimensional governing dynamic equation of energy is accurate for temperature distribution in flow channel but the calculation procedure is complex. The main benefit of using a two dimensional computation is saving time and resources cost, but this advantage is followed by losing the information related to scaling the problem from three dimensions to two dimensions.

In order to obtain the approximation temperature distribution on the both channels of the membrane distillation system, the energy equations were simplified with the following assumptions: (1) laminar flow; (2) symmetrical flow and temperature distribution; (3) no internal generation of energy; (4) both $z$-axis and $x$-axis directions of velocity exists but only $x$-axis velocity is explicitly taken into account, the $z$-axis velocity will be taken into account implicitly as will be demonstrated in resolution algorithm. The equations of energy thus may be obtained as:

$$
\rho C_{p} \frac{\partial T}{\partial t}+\rho C_{p} Q\left(\frac{\partial T}{\partial x}+\frac{\partial T}{\partial z}\right)=k \rho C_{p}\left(\frac{\partial^{2} T}{\partial x^{2}}+\frac{\partial^{2} T}{\partial z^{2}}\right) .
$$


The first step to resolve this PDE (Eq. (4)) is to divide the flow channel into $M$ sub systems in cascade so that the variation of the temperature through the $z$-axe on each sub system is equal to zero. Then, by resolving each sub system separately the summation of flux obtained will give the total pure water flux. The first membrane boundary limit is the inlet flow temperature. The temperatures relaying between the subs systems are the mean temperatures profiles of the previous sub system. The other boundary limits are those in the literature and will be defined in the following. The equation of each sub system is:

$$
\rho C_{p} \frac{\partial T}{\partial t}+\rho C_{p} Q \frac{\partial T}{\partial x}=k \rho C_{p} \frac{\partial^{2} T}{\partial x^{2}}
$$
find:

This PDE is solved using the finite elements and we can

$$
\begin{gathered}
\rho_{a, i} C_{a, p} \frac{\partial T_{a, i}(t)}{\partial t}+\rho_{a, i} C_{a, p} Q \frac{T_{a, i}(t)-T_{a, i-1}(t)}{\Delta x} \\
=k_{a, i} \frac{T_{a, i+1}(t)-2 T_{a, i}(t)+T_{a, i-1}(t)}{\Delta x^{2}}
\end{gathered}
$$

And for the cold side:

$$
\begin{gathered}
\rho_{\text {eau }} C_{b, p} \frac{\partial T_{a, i}(t)}{\partial t}+\rho_{\text {eau }} C_{b, p} Q \frac{T_{b, i}(t)-T_{b, i-1}(t)}{\Delta x} \\
=k_{b, i} \frac{T_{b, i+1}(t)-2 T_{b, i}(t)+T_{b, i-1}(t)}{\Delta x^{2}}
\end{gathered}
$$

The boundary limits used are those of the heat transfer mechanism [17]:

$$
\begin{aligned}
& T_{N+1}(t) \\
& =\frac{1}{3}\left(4 T_{N}(t)-T_{a, N-1}(t)-2 \Delta x \frac{J \lambda+k_{m} \frac{T_{a, N}(t)-T_{b, N}(t)}{\delta_{m}}}{k_{i}}\right)
\end{aligned}
$$

with:

$$
\begin{gathered}
k_{m}=\varepsilon k_{g}+\left(1-\varepsilon k_{s}\right), \\
k_{i}=-0.46+5.8 \times 10^{-3}\left(T_{i}+273.15\right) \\
-7.18 \times 10^{-6}\left(T_{i}+273.15\right)^{2} \text { and } \\
\rho_{a, i}=\frac{100}{\frac{3.5}{\rho_{\mathrm{NaCl}}}+\frac{96.5}{\rho_{\text {eau }}\left(T_{i}\right)}}, \\
\rho_{\text {eau }}\left(T_{i}\right)=819+1.49\left(T_{i}+273.15\right)-0.003\left(T_{i}+273.15\right)^{2} .
\end{gathered}
$$

We obtain then, a matrix $(M \times N)$ of ordinary differential equations (ODE). $M$ is the number of sub system, which the membrane is divided in cascade in the direction of $z$-axis. $N$ is the number of equation, which each sub system is discretized in space in the $x$-axis using finite elements. The diagram in Figure 2 explains the schematic of resolution.

Let put $T_{a, i}(t)=\psi_{i}^{\text {hot }}$ and $T_{b, i}(t)=\psi_{i}^{\text {cold }}, \Sigma_{i}$ can then be described by equations in stat space. Let's take:

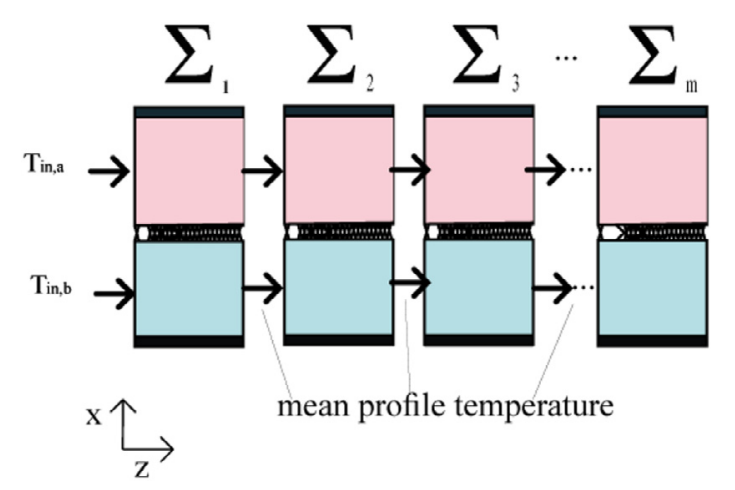

Figure 2. Schematic diagram resolution algorithm.

$$
u_{i}=\sum_{i=1}^{N} \frac{\psi_{i}}{N}=Y_{i-1}
$$

The output of each sub system is the mean profile temperature and it is also the input of the next sub system. We obtain the stat space equation of the sub system $\Sigma_{i}$ in the hot side:

$$
\left\{\begin{array}{c}
\dot{\psi}_{1}^{\text {hot }}=k_{a, 1} \frac{\psi_{2}^{\text {hot }}-2 \psi_{1}^{\text {hot }}+u_{i}^{\text {hot }}}{\rho_{a, 1} C_{a, 1} \Delta x^{2}}-Q \frac{\psi_{1}^{\text {hot }}-u_{i}^{\text {hot }}}{\Delta x} \\
\dot{\psi}_{2}^{\text {hot }}=k_{a, 1} \frac{\psi_{3}^{\text {hot }}-2 \psi_{2}^{\text {hot }}+\psi_{1}^{\text {hot }}}{\rho_{a, 2} C_{a, 2} \Delta x^{2}}-Q \frac{\psi_{2}^{\text {hot }}-\psi_{1}^{\text {hot }}}{\Delta x} \\
\vdots \\
\dot{\psi}_{N}^{\text {hot }}=k_{a, N} \frac{\psi_{N+1}^{\text {hot }}-2 \psi_{N}^{\text {hot }}+\psi_{N-1}^{\text {hot }}}{\rho_{a, N} C_{a, N} \Delta x^{2}}-Q \frac{\psi_{N}^{\text {hot }}-\psi_{N-1}^{\text {hot }}}{\Delta x}
\end{array}\right.
$$

and

$$
\left\{\begin{array}{c}
y_{i}^{\text {hot }}=\frac{1}{N}\left(\psi_{1}^{\mathrm{hot}}+\psi_{2}^{\mathrm{hot}}+\ldots+\psi_{N}^{\mathrm{hot}}\right) \\
u_{i}=Y_{i-1}
\end{array}\right.
$$

and in the cold side we obtain:

$$
\left\{\begin{array}{c}
\dot{\psi}_{1}^{\text {cold }}=k_{a, 1} \frac{\psi_{2}^{\text {cold }}-2 \psi_{1}^{\text {cold }}+u_{i}^{\text {cold }}}{\rho_{a, 1} C_{a, 1} \Delta x^{2}}-Q \frac{\psi_{1}^{\text {cold }}-u_{i}^{\text {cold }}}{\Delta x} \\
\dot{\psi}_{2}^{\text {cold }}=k_{a, 1} \frac{\psi_{3}^{\text {cold }}-2 \psi_{2}^{\text {ocld }}+\psi_{1}^{\text {cold }}}{\rho_{a, 2} C_{a, 2} \Delta x^{2}}-Q \frac{\psi_{2}^{\text {cold }}-\psi_{1}^{\text {cold }}}{\Delta x} \\
\vdots \\
\dot{\psi}_{N}^{\text {cold }}=k_{a, N} \frac{\psi_{N+1}^{\text {cold }}-2 \psi_{N}^{\text {cold }}+\psi_{N-1}^{\text {cold }}}{\rho_{a, N} C_{a, N} \Delta x^{2}}-Q \frac{\psi_{N}^{\text {cold }}-\psi_{N-1}^{\text {cold }}}{\Delta x}
\end{array}\right.
$$

and

$$
\left\{\begin{array}{c}
y_{i}^{\text {cold }}=\frac{1}{N}\left(\psi_{1}^{\text {cold }}+\psi_{2}^{\text {cold }}+\ldots+\psi_{N}^{\text {cold }}\right) \\
u_{i}=Y_{i-1}
\end{array}\right.
$$

with $i=1, M$ and $u_{l}=T_{\text {in }}$ (in the hot and cold side separately).

When seen as a complete system we can write:

$$
T_{a, \text { in }}=u_{1}^{\text {hot }}, T_{b, \text { in }}=u_{1}^{\text {cold }}, T_{a, \text { out }}=y_{M}^{\text {hot }}, T_{b, \text { out }}=y_{M}^{\text {cold }}
$$

and

$$
J=\sum_{i=1}^{M} J_{i}
$$

where $J_{i}$ is the pure water flux in each sub system. 


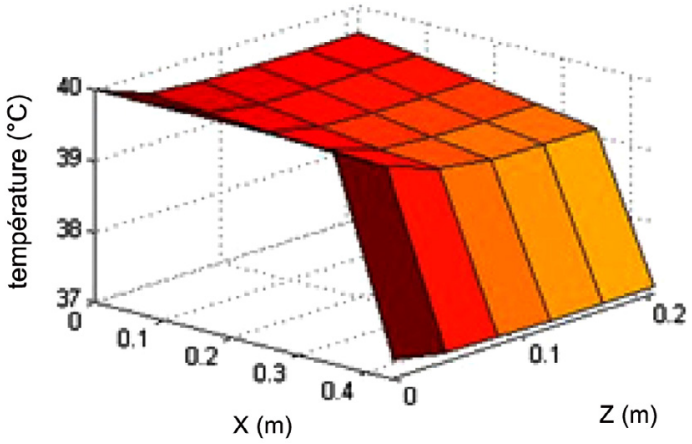

Figure 3. Schematic diagram of hot flow temperature in two dimensions coordinates.

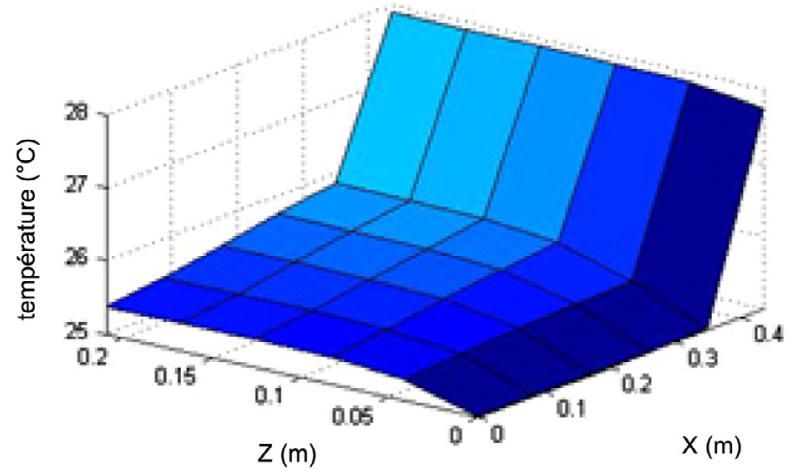

Figure 4. Schematic diagram of cold flow temperature in two dimensions coordinates.

Figures 3 and 4 show the bi dimensional result of temperature equation in the flow channels of membrane. As can be seen, the temperature in the hot side decrease along the $x$ and $z$-axis of membrane, in the same way the cold side temperature increase along the $x$ and the $z$-axis, this phenomenon is called polarization.

A case study from literature has been investigated. The data used in our simulations like geometry, physical properties and operating conditions are the same used in [4]. Simulations have been conducted for different inlets temperature. Compared with the stationary experimental data in [4].

As shown in Figure 5, the computed mass flux densities are in good agreement with the measurements.

\section{Nonlinear observer}

In practice, only the inlet and outlet temperatures are accessible for measurement. The need of nonlinear observer arises. The observer will estimate all temperatures inside the membrane and from those temperatures it will be possible to estimate polarization coefficient, polarization ratio, and pure water flux. Because the structure in cascade of the model developed in the previous section, a nonlinear unknown input observer (NUIO) is used. NUIO are used for different systems,

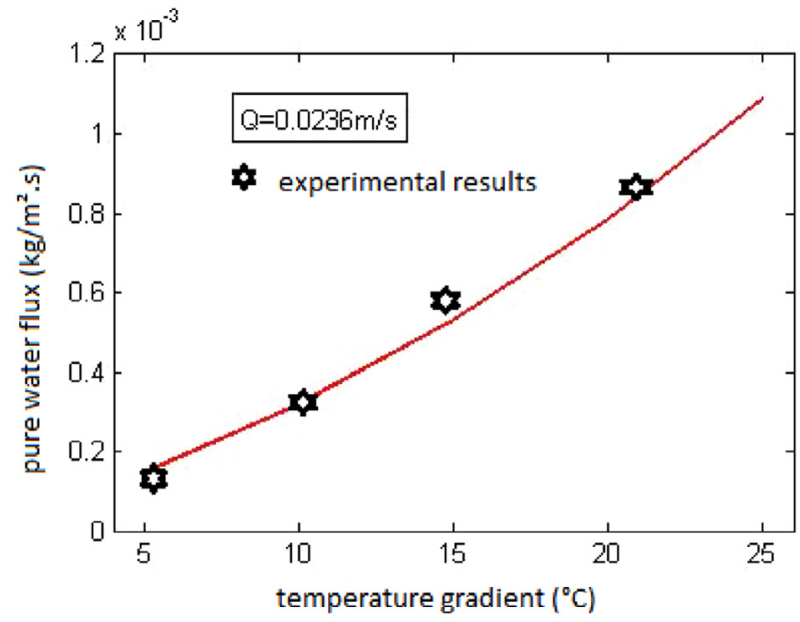

Figure 5. Pure water flux function of temperature gradient.

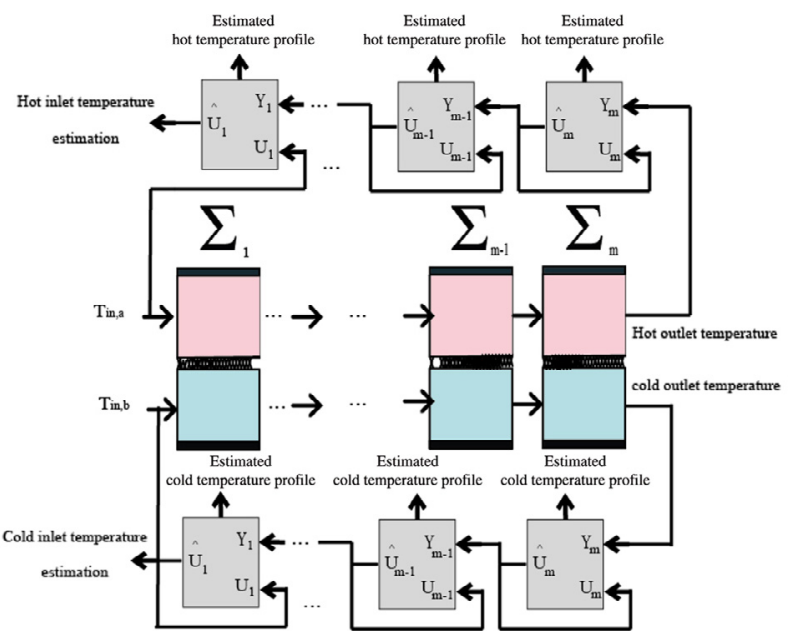

Figure 6. Schematic diagram the nonlinear unknown input observer (NUIO) in DCMD process.

a design of unknown input functional observers for nonlinear systems with application to fault diagnosis has been presented in [18]. A nonlinear unknown input observer design for diesel engines is described in [19]. In [20] an observer-based residual design for nonlinear systems with unknown inputs is developed. The structure of the NUIO in our study is in cascade. The advantage of this kind of structure is that we can increase the accuracy of the model by increasing the number of subsystems describing the membrane. By Applying the NUIO to the last subsystem we can estimate all temperatures inside it. We can also estimate the unknown input of these sub system, which is the output of the previous subsystem. Using this output we can estimate the temperatures inside the previous subsystem. Using this method, we can ascend to the first subsystem, which has his input accessible for measurement (inlet temperature). The following scheme (Figure 6) describes the observer used. 
This structure has a lot of advantages; it is possible to increase the accuracy of the model by increasing the number of subsystems. Also, when estimating all temperatures inside the membrane, we will be able to calculate the estimated flux in each part of the membrane, calculate the total flux, and then, calculate different parameters such as polarization ratio and polarization coefficient.

\subsection{Development}

The NUIO is an observer based on a model, the structure of the hot side observer of the subsystem $i$ is:

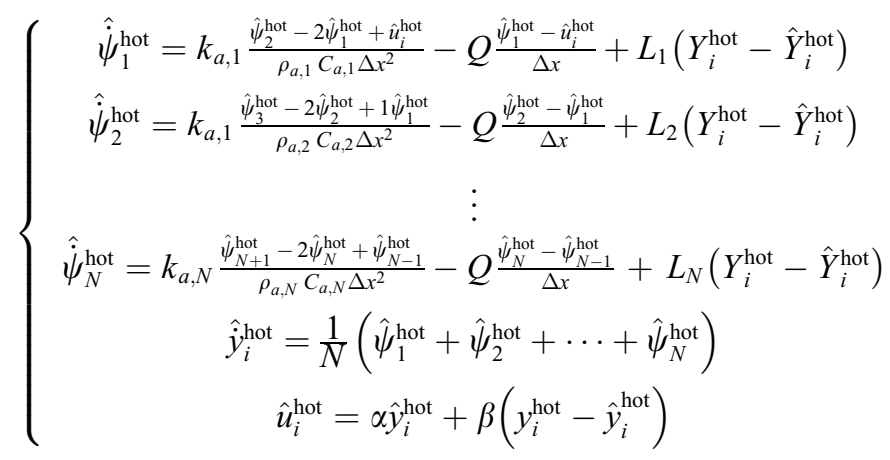

and $y_{i}$ is the outlet temperature, which is accessible for measurement. Because of the structure of the model in cascade, the estimated unknown input is the output of the previous subsystem $\hat{u}_{i}=y_{(i-1)}$ this output can be used to estimate all parameters of the previous subsystem $(i-1)$ and by continuing with same principal to the first subsystem we can estimate all the parameters of the membrane. The same development is applied to the cold side:

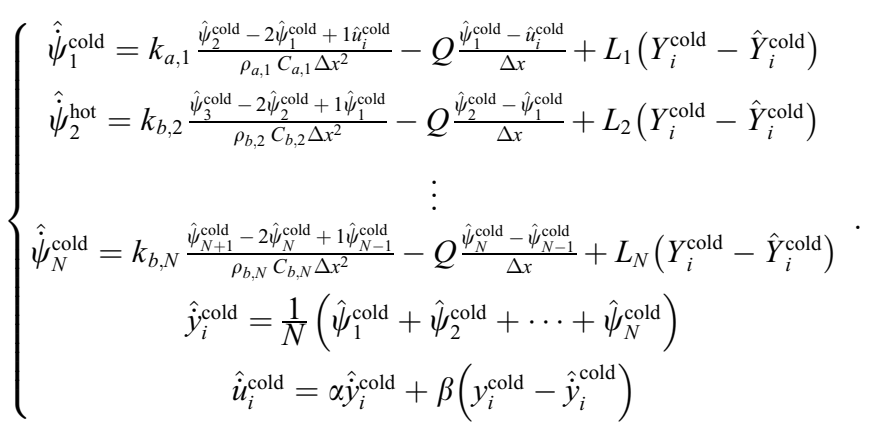

\section{Simulations results}

\subsection{Estimation of the pure water flux}

The pure water flux is accessible for measurement; it is a good parameter to evaluate the accuracy of the observer by plotting the estimation error between the measured flow and the estimated flow given by the NUIO. The estimated pure

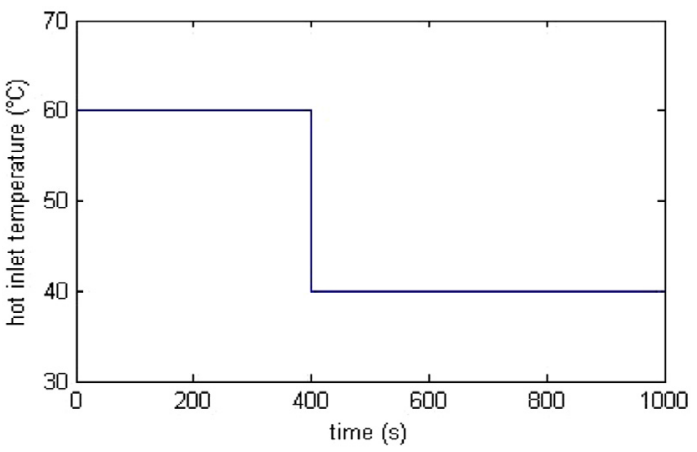

Figure 7. Step of hot inlet temperature introduced at $t=400 \mathrm{~s}$.

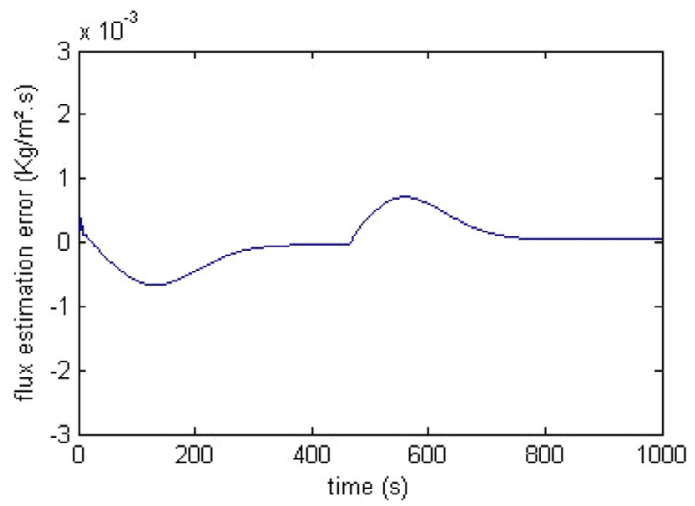

Figure 8. Pure water flux production estimation error.

water flux is given by the following equation (17):

$$
\hat{J}=c_{m}\left(\left(1-x_{\mathrm{NaCl}}\right)\left(1-0.5 x_{\mathrm{NaCl}}-10 x^{2}{ }_{\mathrm{Nacl}}\right) \hat{P}_{a}^{\mathrm{sat}}-\hat{P}_{b}^{\mathrm{sat}}\right)
$$

with

$$
\hat{P}_{k}^{\text {sat }}=133.322 \times 10^{\left(8.10765-\left(1450.286 /\left(\hat{T}_{k}+235\right)\right)\right)}, \quad k=a, b .
$$

By introducing a step in hot water inlet (Figure 7), the pure water flux changes, this is due to the predominance of the temperature gradient in pure water production. The observer follows this changes and the estimating error is given in Figure 8.

We can see that the observer gives a good estimation of the pure water flux, and can be used for fault diagnosis to detect abnormal pure water production.

\subsection{Estimating polarization ratio}

Because temperature polarization is the dominant effect on the pure water flux through membrane, the analysis of temperature polarization phenomenon is presented in Figure 9.

As seen from Figure 9, results show that the temperature polarization ratio, which is defined as $T_{a, N+1} / T_{a, 0}$ or $T_{b, N+1} /$ $T_{b, 0}$, increases as the hot stream proceeds in the flowing direction. This increase is due to decrease of the heat-transfer coefficient in the flow channel, resulting in increasing both the velocity boundary layer and thermal boundary layer. 


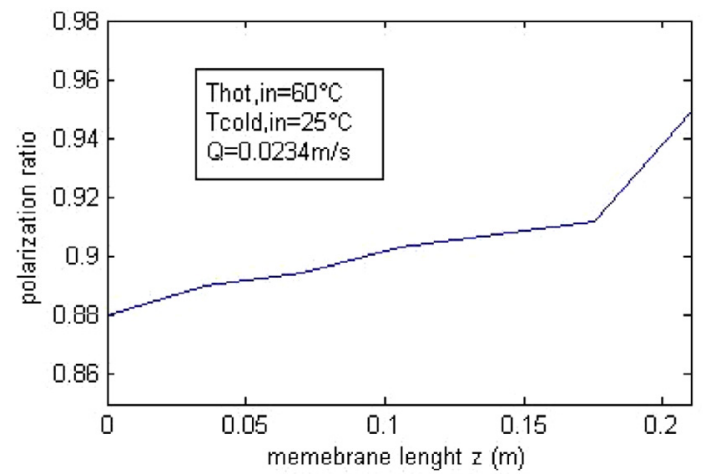

Figure 9. Polarization ratio in the longitudinal axe of the membrane.

\subsection{Estimation of the polarization coefficient}

Temperature polarization characterizes the temperature distribution between bulk stream $\left(T_{a, 0}-T_{b, 0}\right)$ and membrane interface temperatures, $\left(T_{a, N+1}-T_{b, N+1}\right)$, which mainly depends on the ratio of heat transfer in the flow channels and through the membrane. The coefficient characterizing this relation is the temperature polarization coefficient:

$$
\tau=\frac{T_{a, N+1}-T_{b, N+1}}{T_{a, 0}-T_{b, 0}} .
$$

It can be seen that, for a very efficient heat transfer between bulk streams and membrane interfaces $\left(T_{a, N+1} \approx T_{a, 0}\right.$ and $\left.T_{b, N+1} \approx T_{b, 0}\right)$, the coefficient $\tau$ approaches 1 . In the case of low heat transfer coefficients in the channels, compared to heat transfer coefficient through the membrane $\left(T_{a, N+1} \approx T_{b, N+1}\right)$, the polarization coefficient $\tau$ approaches 0 . In MD systems, $\tau$ mainly depends on channel set-up, module design and operation. The temperature polarization coefficient increases with an increasing heat transfer coefficient, conversely, an increase of the mean temperature, which leads to an increase of vapor transport can also lead to significant reductions in temperature polarization coefficients [21]. Values below 0.1 for flat membranes in laminar flow operation, and above 0.9 for narrow hollow fiber membranes in turbulent flow configuration have been reported [22]. The Figure 10 shows the temperature polarization coefficient estimating error inside the membrane at precise longitudinal position.

We can see also from Figure 10 that the observer gives a good estimation of the polarization coefficient compared with the estimation given by the model. The temperature polarization coefficient must be taken into account in order to calculate the mass transfer from the bulk temperatures, which are usually well known, compared to the membrane interface temperatures, which are almost immeasurable. It can be seen that temperature polarization has a significant influence on flux and on the overall process efficiency, and therefore should be the focus of the MD process design.

\subsection{Estimation of the inlet temperature}

The observer developed is an observer with an unknown input observer, and the input is estimated using a model.

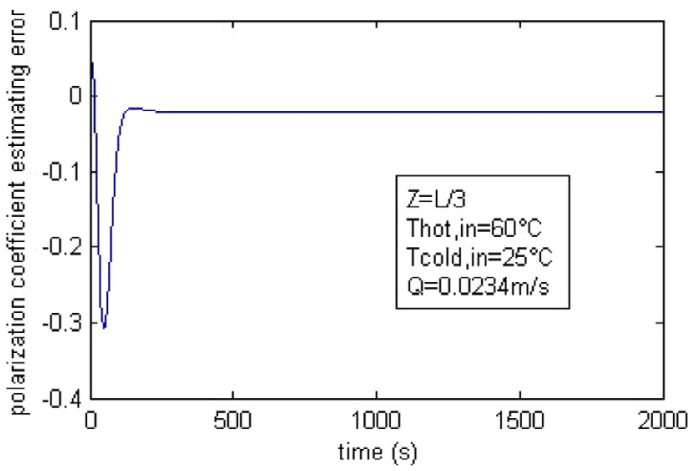

Figure 10. Polarization coefficient estimating error inside DCMD.

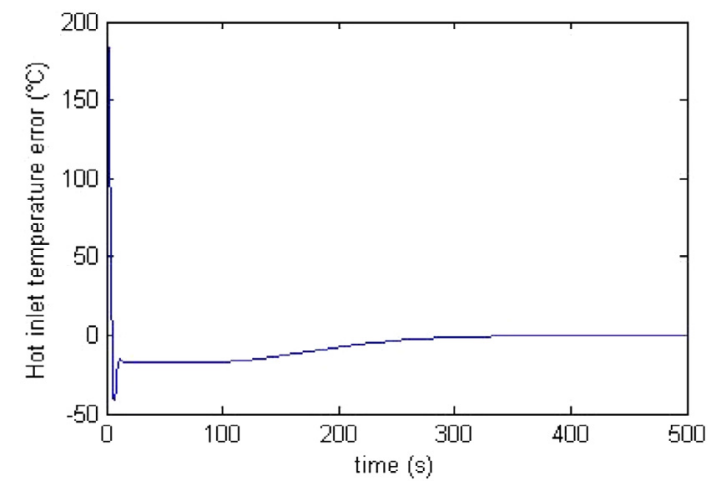

Figure 11. Hot inlet estimating error.

Because of the structure in cascade of the observer, we have to estimate the inputs of all subsystem. The model used in our study is given in the following:

$\hat{u}_{i}^{\text {hot }}=\alpha \hat{y}_{i}^{\text {hot }}+\beta\left(y_{i}^{\text {hot }}-\hat{y}_{i}^{\text {hot }}\right)$ with $\alpha>1$ for the hot side and, $\hat{u}_{i}^{\text {cold }}=\alpha \hat{y}_{i}^{\text {cold }}+\beta\left(y_{i}^{\text {cold }}-\hat{y}_{i}^{\text {cold }}\right)$ with $\alpha<1$ for the cold side.

Because the first input (hot and cold inlet temperature) is accessible for measurement, it is important to validate the model of the input by plotting the estimation error (Figure 11) between the estimated input and the measured input.

We can see that the first input is estimated correctly, and this input is the most difficult to estimate, because the estimation error is cumulative. For accuracy reasons, the first subsystem of the observer is fed with the real measured input.

\subsection{Estimation of the longitudinal temperature distribution}

The two-dimensional model is useful to study the transport mechanism across the micro porous membrane in detail. The membrane surface temperature distributions in the flowing direction are shown in Figure 12. Hot side decrease along flowing direction while the membrane surface temperature of cold side, increase with increasing longitudinal coordinate. Furthermore, the trans membrane pressure affected by the temperature gradient between both membrane surfaces taper along the 


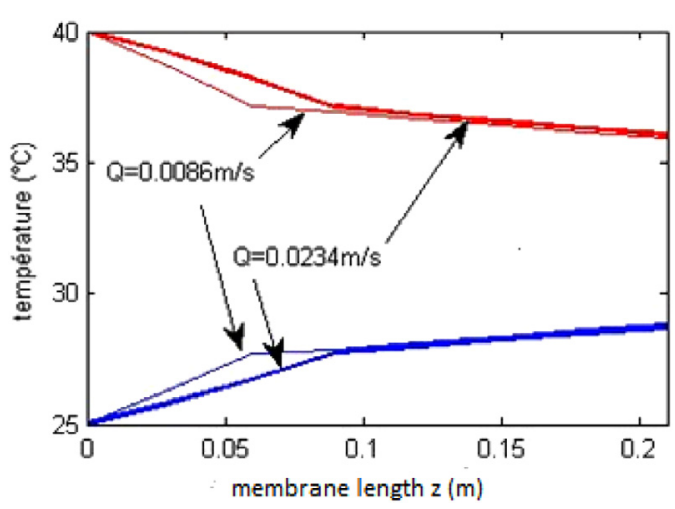

Figure 12. Schematic diagram of temperature along $z$ axes.

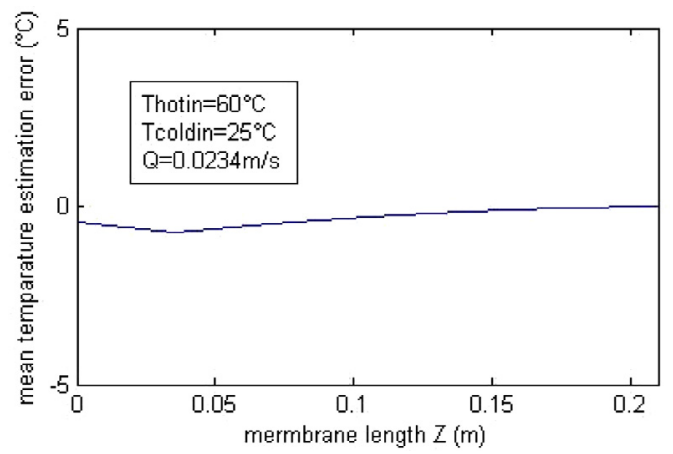

Figure 13. Longitudinal temperature estimating error along DCMD.

flowing direction, resulting in decreasing the pure water productivity per unit area of membrane. Accordingly, the pure water flux decreases along the flowing direction due to the temperature difference decreasing. Though increasing the volumetric flow rate is helpful to enhance the productivity of pure water as confirmed from Figure 12.

A comparison with the results given by the observer and those given by the model is shown in Figure 13.

The results show that the estimation error increases in the opposite of the flow direction. This is due to the configuration of the observer, which starts estimations from the last subsystem. The estimation error continues to increase until the introduction of the real measurement of the inlet temperature. The estimation error then decrease until the first input estimation which is measurable.

\section{Conclusion}

In this paper, a dynamic bi dimensional model has been proposed, a resolution algorithm has been proposed to solve the PDE and simulation results are given. The model is implemented using Matlab/Simulink. A nonlinear observer is used to estimate the major phenomenon inside the membrane. Results show that the model proposed gives good prediction of pure water production. Also, the observer gives a good prediction of the temperatures and flux inside the membrane.
The observer can be used to monitor the membrane and the results can be used for fault diagnostic, which is a major step in this kind of MD process. We will continue our efforts to build an experimental setup so that we can perform experiments on our model and observer.

\section{References}

1. Lawson KW, Lloyd DR. 1996. Membrane distillation. II. Direct contact MD. Journal of Membrane Science, 120, 123-133.

2. Koschikowski J, Wieghaus M, Rommel M. 2003. Solar thermal-driven desalination plants based on membrane distillation. Desalination, 156, 295-304.

3. de Andrés MC, Doria J, Khayet M, Pena L, Mengual JI. 1998. Coupling of a membrane distillation module to a multieffect distiller for pure water production. Desalination, 115, 71-81.

4. Chen T, Ho C, Yeh H. 2009. Theoretical modeling and experimental analysis of direct contact membrane distillation. Journal of Membrane Science, 3(30), 279-287.

5. Ashoor BB, Fath H, Marquardt W, Mhamdi A. 2012. Dynamic modeling of direct contact membrane distillation processes. Proceedings of the 11th International Symposium on Process Systems Engineering, 15-19 July, Singapore.

6. de la Calle A, Bonilla J, Roca L, Palenzuela P. 2014. Dynamic modeling and performance of the first cell of a multi-effect distillation plant. Applied Thermal Engineering, 70(1), 410-420.

7. Kim Y-D, Thu K, Ghaffour N, Ng KC. 2013. Performance investigation of a solar-assisted direct contact membrane distillation system. Journal of Membrane Science, 427, 345-364.

8. Saif M. 1993. A disturbance accommodating estimator for bilinearsystems. IEEE American Control Conference (ACC 93), June 2-4, San Francisco, CA, USA.

9. Frank PM, Ding X, Guo L. 1990. Nonlinear observer design via extended observer canonical form. Systems and Control Letters, 15, 313-322.

10. Seliger R, Frank PM. 1991. Robust component fault detection and isolation in nonlinear dynamic system using nonlinear observers, Proceedings of the IFAC/IMACS Symposium SAFE PROCESS, Baden-Baden, Germany.

11. Lawson KW, Lloyd DR. 1997. Membrane distillation. Journal of Membrane Science, 124, 1-25.

12. Felder RM, Rousseau RW. 2000. Elementary principles of chemical processes, 3rd ed., John Wiley \& Sons: New York.

13. Schofield RW, Fane AG, Fell CGD. 1990. Gas and vapor transport through microporous membrane. Journal of Membrane Science, 53-159.

14. Iversen SB, Bhatia VK, Dam-Jphasen K, Jonsson G. 1997. Characterization of microporous membranes for use in membrane contactors. Journal of Membrane Science, 130, 205-217.

15. Incropera FP, Dewitt DP. 1996. Fundamentals of heat and mass transfer, 4th ed., John Wiley \& Sons: New York.

16. Bird RB, Stewart WE, Lightfoot EN. 2007. Transport Phenomena, 2nd ed., John Wiley \& Sons: New York.

17. Alklaibi AM, Lior N. 2004. Membrane-distillation desalination: status and potential. Desalination, 171, 111-131.

18. Teh PS, Trinh H. 2013. Design of unknown input functional observers for nonlinear systems with application to fault diagnosis. Journal of Process Control, 23, 1169-1184. 
19. Boulkroune B, Djemili I, Aitouche A, Cocquempot V. 2013. Nonlinear unknown input observer design for diesel engines. American Control Conference (ACC), June 17-19, Washington, DC, USA, pp. 1076-1081.

20. Teh PS. 2011. Observer-based residual design for nonlinear systems with unknown inputs. Australian Control Conference, 10-11 November, Melbourne, Australia, 198-204.
21. Sherwood TK, Pigford RL, Wilke CR. 1975. Mass transfer, New York, McGraw-Hill.

22. Schofield RW, Fane AG, Fell CJD. 1987. Heat and mass transfer in membrane distillation, Journal of Membrane Science, 33, 299-313.

Cite this article as: Khoukhi B, Tadjine M \& Boucherit MS: Nonlinear observer to estimate polarization phenomenon in membrane distillation. Int. J. Simul. Multisci. Des. Optim., 2015, 6, A4. 